population par l'immigration pour pouvoir tirer le meilleur parti de ses richesses.

Les colons européens se dirigeront de préférence vers les états du Sud. Ceux-ci ont encore une très grande capacité d'absorption. Sao Paulo dont la superficie est six fois celle de la Suisse n'a pas encore huit millions d'habitants; il y en a encore moins dans les autres états, Minas (plus grand que la France), Parana (égal à la moitié de l'Italie), Santa Catarina (deux fois la Suisse), Rio Grande do Sul (aussi grand que l'Italie). ${ }^{9}$ Quant aux régions tropicales, elles se peupleront par des migrations internes, car les personnes nées au Brésil, même dans le Sud, sont plus aptes à en supporter le climat.

Principales sources utilisées:

1. Blumenauer Volkskalender für das Jahr 1935. Blumenau 1934.

2. Carta Geographica do Brasil organisada pelo Club de Engenharia do Rio de Janeiro, 1:275000. 1922.

3. DENIS, P.: Le Rrésil au XXe siècle. Paris 1909.

- Amérique du Sud I. Paris 1927.

4. Diccionario Chorographico Brasileiro. Rio de Jareiro 1929.

5. FREISE, F.: Brasiliens Bevölkerungskapazität. Petermanns Mitteilungen, 82, 1936, S. $143-147$.

6. FREYRE, G.: Casa Grande e Senzala. Rio de Janeiro 1936.

7. GUTERSOHN, H.: Sao Paulo. Vierteljahresschrift der Naturforschenden Gesellschaft Zürich, 85, 1940, S. 149-255.

- Das Kemland von Minas Gerais. Arbeiten Geographisches Institut Eidgenössische Technische Hochschule Zürich. Nr. $3,1945$.

8. JAMES, P.: Latin America. New York 1942.

9. LUFFT, H.: Lateinamerika. Leipzig 1930.

10. MAURETTE, F., ec SIEVERS, E.: L'Immigration et la Colonisation au Brésil. en Argentine et en Uruguay, I, Brésil. Revue Internationale du Travail, 35, 1937, p. 230-263.

11. METTLER, A. : Les factcurs géographiques dans la découverte et l'occupation du Brésil. Annales de Géographie, 46, 1937, p. 61-75.

12. Ministerio das Relaçoes Exteriores: Brasil 1940-1944. Rio de Janeiro.

13. PAUWELS, P.: Aclas Geographico Geral e especialmente do Brasil, Sao Paulo, Cayciras. Rio de Janeiro 1936.

11. PENCK, A.: Das Hauptproblem der physischen Anthropogeographie. Sitzungsberatungen der Preussischen Akademie der Wissenschaften 22, 1924, S. 242-257.

13. SCHMIEDER, O.: Länderkunde Südamerikas. Leipzig und Wien 1932.

Plusieurs de ces ouvrages en particulier les numéros $3,7,8,15$, contiennent des bibliographies détaillées.

- Selon des évaluations au 1 janvier 1944, la population de ces états était la suivante: Sao Paulo, 7733500; Minas Gerais, 7310000; Parana, 1279700; Santa Catarina, 1255900; Rio Grande do Sul, 3578600.

\title{
ZENTRALINDISCHE FRUCHTBARKEITSRITEN UND IHRE BEZIEHUNGEN ZUR INDUSKULTUR
}

\author{
Von Wilhelm Koppers
}

Mit 8 Abbildungen

\section{Einleitung}

$\mathrm{Da}$ der Aufdeckung der Induskultur für weite Bereiche der Indienforschung eine umwälzende Bedeutung zukommt, wird heute von den maßgebenden Wissenschaftern allgemein anerkannt. Wenn Sir MARSHALL schon vor fünfzehn Jahren darauf hingewiesen hat, daß eine Anzahl der Wesenselemente des heutigen Hinduismus auf die Induskultur zurückgeführt werden muß, so hat die Forschung der nachfolgenden Zeit dieses Resultat nicht nur bestätigen, sondern um manche bedeutsame Punkte erweitern können ${ }^{1}$. Vom Standpunkte bestimmter Fruchtbarkeitsriten aus, die ich selbst in Zentralindien bei den Primitivstämmen der Bhil und der Gond zu beobachten Gelegenheit hatte, ergibt sich, wie ich meine, wenn nicht eine sichere, so doch eine wahrscheinliche neue Beziehung zu bestimmten, und zwar bis jetzt dunkel und ungeklärt gebliebenen Funderscheinungen der Induskultur hin. Den Fruchtbarkeitsritus, um den es

1 J. MARSHALL: Mohenjo-Daro and the Indus Civilization. I., London 1931, S. 110 f. Vgl. R. O. EHRENFELS: Motherright in India. Hyderabad (Deccan) 1941, S. $180 \mathrm{ff}$. 
sich in diesem Falle bei den Bhil und den Gond handelt, repräsentiert die Kali-DurgaNovene. Was also dabei in Frage steht, muß zunächst kurz vorgeführt werden.

\section{Die Kali-Durga-Novene bei den Bhil}

Um Mißverständnissen vorzubeugen, ist zu bemerken, daß der Kali-Durga-Kult in Indien namentlich in Hindu-Kreisen eine weite Verbreitung hat. Dasselbe gilt von der Novene, die dieser Göttin zu Ehren veranstaltet wird ${ }^{2}$. Doch von all dem sehen wir hier ab und richten unsern Blick auf die Art der Feier dieser Novene bei den Bhil und den Gond.

Wie ähnlich sonstwo, so wird auch bei den Bhil die Kali-Durga-Novene mit verschiedenen Namen bezeichnet. Man spricht von den neun Zwârâ-Tagen. Zwârâ (Hindi ja wârâ) heißt Gerste. Die Novene trägt diesen Namen mit Rücksicht auf die Gerste, die bei dieser Gelegenheit gesät wird. Das Nähere darüber werden wir bald kennenlernen. Ein anderer Name ist Nortân (Hindi Navartân oder Navrâtra), was neun Nächte bedeutet. Diese Benennung knüpft an die Tatsache an, daß die Veranstaltung vornehmlich zur Nachtzeit sich abwickelt. Die Bhil feiern die Novene gegen Ende der Regenzeit. Im Jahre 1938 z. B. begann sie am 24. September. Sie wird übrigens so gelegt, daß am Tage nach Schluß, also am 10. Tage, das Dâharo-Fest fällt, welches mit dem allgemein indischen Dashahéra ( $d$ das» heißt zehn) identisch ist. Die Feier der Novene kann auch auf Grund eines Gelübdes vorgenommen werden. In diesem Falle ist sie natürlich nicht an einen bestimmten Termin gebunden.

Wie dem im einzelnen auch sei, auf jeden Fall gibt sich die Kali-Durga-Novene als besondere Angelegenheit des Zauberers (bei den Bhil Barwo genannt) zu erkennen. Der Zauberer vor allem tritt dabei in Tätigkeit, und während sonst allgemein die Klienten für die Opfertiere aufzukommen haben, werden sie in diesem Falle vom Zauberer selbst beigestellt und am Schlusse von allen Dorfgenossen gemeinsam verzehrt.

Die Dorfgemeinschaft errichtet eine kleine Hütte, in welcher der Zauberer die neun Tage zu verbringen hat. Am Abend des ersten Tages versammelt sich alles vor dieser Hütte. Der Zauberer hat sein Zaubertischlein bereitstehen. Zunächst träufelt der Dorfvorsteher davor einige Tropfen Daru auf den Boden, und zwar zu Ehren der Erdgöttin, der Zami Mata. Das gleiche tut dann auch der Zauberer selbst. Hierauf bedeckt dieser das Tischlein mit Erde und sät Weizen- und Gerstenkörner hinein, etwa 2/3 Weizen und $1 / 3$ Gerste. Der Einfachheit willen wird im folgenden immer nur von Weizen und Weizengärtchen die Rede sein.

Im Wachsen dieses Weizens erblicken die Bhil, wie sie selber sagen, ein Vorzeichen für das gute Gedeihen der bevorstehenden zweiten, d. h. der Winterernte, die vor allem, wenigstens für Teile des Bhil-Gebietes, eine Weizenernte ist. Anderseits ist zu beachten, daß nach Auffassung der Bhil nicht so ausgesprochen die Kali-Durga im Hintergrund der Veranstaltung steht. Die Novene soll vielmehr an die Gesamtheit der höheren Wesen und Gottheiten gerichtet sein. Dieser Umstand ist nicht unwichtig. Er gibt zu erkennen, wofür übrigens auch andere Anzeichen sprechen, daß der KaliDurga-Kult an und für sich nicht eine für sie typische Angelegenheit darstellt. Dieses findet schließlich seine Bestätigung auch noch darin, daß keiner von den Teilen, in die der Zauberer sein Weizengärtchen zerlegt und die er einzelnen verschiedenen Gottheiten widmet, der Kali-Durga als solcher zuerkannt wird.

Der Zauberer teilt das Gärtchen in vierzehn Anteile auf. Eines gehört der KazalKönigin, die eine der vielen Muttergottheiten darstellt ${ }^{3}$. Ihr Teil befindet sich in einem kleinen irdenen Gefäß, das auf die Oeffnung eines irdenen Wasserkruges gestellt wird.

So z. B. H. v. GLASENAPP: Der Hinduismus. München 1922, S. 355.

- Kazali-Rani (Kazal-Königin) = Kazali-Mata; Kazal heißt die aus Ruß und Oel hergestellec Farbe, welche Fraucn und Mädchen sich wohl um die Augen reiben, zum Schutze gegen den bösen Blick und auch, um so, wic man glaubt, schön zu erscheincn. 


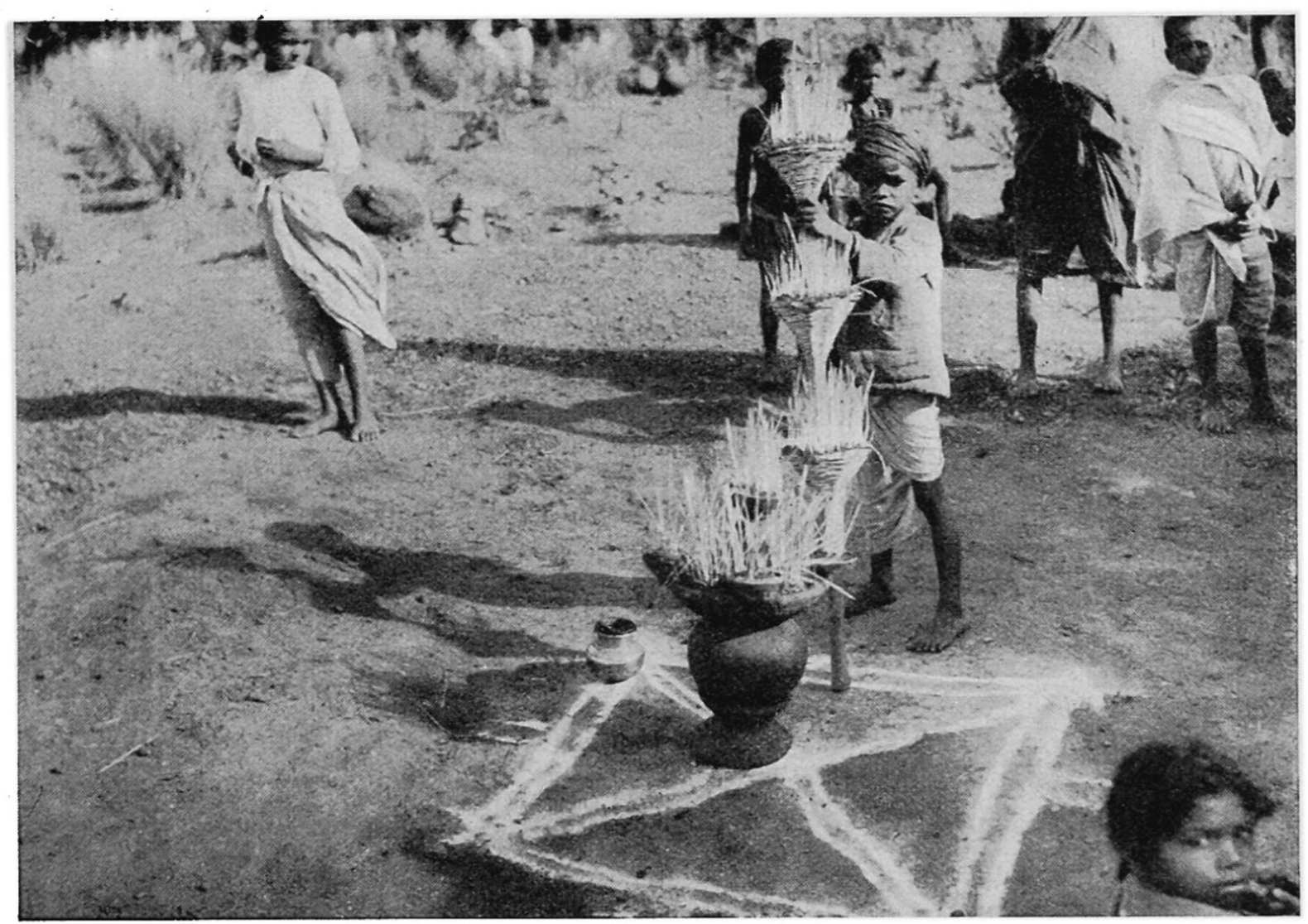

Abb. 1. Gond. Töpfe und Dambusständer weıden auf das magische Viereck (Chauk) gestellt. Photo Koppers

Ein anderer Teil gilt den «kleinen bösen Geistern», worunter allgemein die Seelen der Verstorbenen verstanden werden; man nennt sie Bhuts. Die zwölf restlichen Partien verteilen sich auf sieben Götter und fünf Göttinnen. Die sieben Götter sind: Kasumor, Wagazo, Ghorazo, Nârhing, Manâto, Kalo Bheru, Dewro. Die Namen der fünf Göttinnen lauten: Sâwan Mata, Zami, Hunwara, Pâthwara, Kâlka ${ }^{4}$.

Während der neun Tage lebt also der Zauberer in der kleinen Hütte und bewässert vor allem seinen Weizen. Abends versammeln sich bei ihm die Leute des Dorfes. Man tanzt und singt bis tief in die Nacht hinein die sogenannten Garbo-Lieder, auf die wir aber hier nicht näher eingehen können ${ }^{5}$.

Die Tage der Novene gelten in besonderer Weise auch als eine Zeit der Initiation für junge Zauberer-Kandidaten. Neun Tage lang hintereinander ist da Gelegenheit, sich im Herumschlagen des Kopfes zu üben, um in Trance-Zustand hineinzukommen und von der Mata und anderen Gottheiten und Geistern ergriffen und besessen zu werden. Viele versuchen es, so auch einmal einen Blick in die außernatürliche Welt des Zauberers zu tun. Die Väter sind vielfach stolz auf die Leistungen ihrer Buben. Aber zu wirklichen und angesehenen Zauberern entwickeln sich - glücklicherweise -, so möchte man sagen, nur wenige.

Am neunten Tage der Novene findet die Hauptfeier statt. Mittels Mehl wird auf dem Boden ein Sok (im Hindi Chauk ${ }^{6}$, was Zauberviereck bedeutet) gezeichnet. Darüber werden mit aller Sorgfalt trockene Holzstäbchen gelegt, die man anzündet. Gleich-

- Diese Götter und Göttinnen finden sich alle wieder in der sogenannten großen Krankheitszaubcrei. Vgl. L. JUNGBLUT: Magic Songs of the Bhils. Internationalcs Archiv für Ethnographic, 43, 1941.

- Dic Garbo-Lieder behandle ich etwas cingehender in meinem Buche "Der Dschungelleute Freud und Leid», Las hef.cretlich in nicht allzuferncr Zeit erscheinen kann.

- Die magischen Kreisc (mandala) und Achnliches spielen im Tantrismus-Saktism.us eine große Rollc. In dicsclte Rcitc gehört der Chauk (Bhili, sok), das Zaubervicreck. 
zeitig entfaltet die Zaubertrommel, die.Doppelschädeltrommel oder auch Sivas Trommel, ihre laute und lärmende Tätigkeit. Die Adepten des Zauberers fangen an, mit dem Kopf wie wild herumzuschlagen. Schließlich stellen sich alle um das Feuer und werfen sich, am ganzen Leibe zitternd, mit bloßem Körper darauf und löschen es aus. (So sah Missionar Jungblut es in Jhabua ${ }^{7}$.) Andernorts indes (z. B. in Rambhapur ${ }^{8}$ ) wurde das Feuer mit bloßen Füßen ausgetreten.

Unterdessen hat der Zauberer die Vorbereitung zu einer Prozession zum Fluß oder zur Wasserstelle getroffen, wo der Weizen dem nassen Element anvertraut wird. «Den Garten sinken oder erfrischen machen", wie die Bhil sagen. Der Weizen, der dank der sorgfältigen Pflege und Bewässerung schon etwa $20-30 \mathrm{~cm}$ hochgewachsen ist, wird samt der anhaftenden Erde vom Zauberer auf mehrere Körbchen aufgeteilt. Von einzelnen Teilnehmern wird je ein Körbchen entgegengenommen und getragen. Ein unverheiratetes Mädchen aber trägt den Wasserkrug, auf dem der Weizen der «kleinen bösen Geister» stand. Der Prozession voran wird eine Art Standarte getragen. Die Prozessionsordnung ist im übrigen die folgende:

Die Standartenträger schreiten an der Spitze. Hierauf folgen die Leute, welche die großen Trommeln bedienen. An dritter Stelle kommt der Zauberer mit seinen Lehrlingen. Es wird eifrig mit dem Kopf hin und her geschlagen, und auf die "Besessenen» sausen manche Schläge mit aus Hanfstricken verfertigten Geißeln, wie auch mit Ketten, nieder ${ }^{9}$. Die darauffolgende vierte Gruppe wird von den Trägern der Teile des Gärtchens gebildet. Daran schließt sich das Volk an, worunter auch das Mädchen sich befindet, das jenes Wassergefäß, von dem die Rede war, auf dem Kopfe trägt. Den Schluß bildet der Dorfvorsteher. Er trägt das blanke Schwert in der Hand.

Unterwegs soll manchmal der eine oder andere der "Zauberlehrlinge», von der Bhowani-Mata ergriffen, an der Standartenstange emporklettern. Bhowani heißt Zauberin. Unter diesem Namen ist die Kali-Durga auch unter den Bhil, wie überhaupt im westlichen Indien, ziemlich gut bekannt.

An der Wasserstelle angekommen, träufelt der Zauberer zunächst ein wenig Daru auf den Boden. Darauf nehmen seine Gehilfen die Gefäße mit Weizen entgegen und versenken ihn samt der Erde bis aut den Grund des Wassers hinab. Das Mädchen, welches das Wassergefäß trug; leert dasselbe und füllt es an Ort und Stelle mit neuem Wasser. Darauf werden die Helden des Tages (der Zauberer und seine Schüler) von ihren Angehörigen, zumal von den Schwiegereltern, falls sie bereits solche haben, beschenkt.

Hierauf zieht alles wieder ab. Die Frauen gehen nach Hause, das Männervolk aber und das Mädchen mit dem Krug voll neuen Wassers kehren zu der kleinen Hütte des Zauberers zurück. Dort angekommen, stellt das Mädchen den Krug nieder und entfernt sich ebenfalls, so daß jetzt nur männliche Individuen zurückbleiben. Der Zauberer fordert die Anwesenden auf, nun das «Kaleh»-Wasser zu trinken. Er gibt einem jeden eine Handvoll davon. Dieses Wasser heißt auch matrélun pani, bespruchtes Wasser. $\mathrm{Da}$ vorher alle Frauen weggeschickt werden, geschieht deshalb, um zu verhindern, daß eine Frau, durch den Genuß dieses «Götterwassers» gestärkt, zur Hexe werden und Schaden anrichten könnte.

Nach Beendigung auch dieser letzten Zeremonie schließt die Novene mit einem allgemeinen Essen und Trinken, für das ja der Zauberer selbst die nötige Vorsorge getroffen hat. Je mehr er bietet, desto größer ist sein Name. An diesem Mahl nehmen auch die Frauen teil.

\footnotetext{
? Jhabua ist die Hauptstadt des kleinen Eingeborenenstaates gleichen Narr.ens in Zentralindien. Der weitaus grëßere Teil der Bevölkerung setzt sich hicr aus Bhil zusammen.

- Rambhapur, ein ctwas größerer Ort im Staate Jhabua.

- Diese Geißelungen und Peinigungen bilden die charakteristischen Zugaben bei Veranstaltungen, welche die Zauberer zu Ehren der Kali-Durga durchfübren.
} 


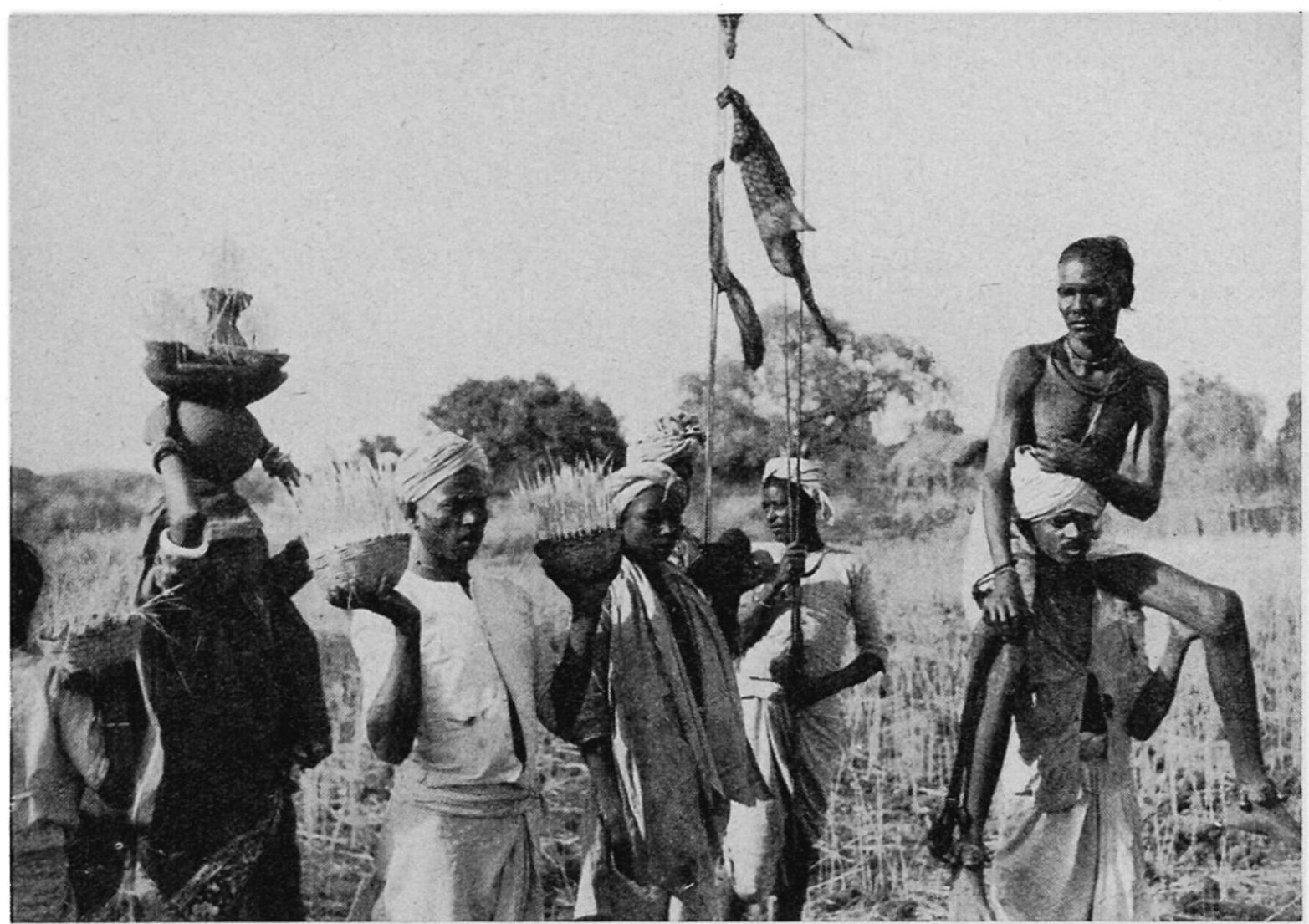

Abb. 2. Gond. Szene aus der zur Wasserstelle ziehenden Prozession. Photo Koppers

\section{Die Kali-Durga-Novene bei den Gond}

Bei den Gond, die in den Maikal-Bergen (etwa 100 Meilen östlich von Jubbelpur, im Quellgebiet der Narbada) wohnen, erfreut sich die Veranstaltung der Kali-DurgaNovene einer besonderen Beliebtheit. Weniger ist das, wie ElwrN bezeugt, bei den Baiga der Fall ${ }^{10}$. Das ist von Bedeutung, weil die Baiga, den Gond gegenüber, an Ort und Stelle die primitivere und ältere Bevölkerung darstellen. Statt $Z$ wârâ gebraucht man bei den Gond zur Bezeichnung der Novene das Hindi-Wort für Gerste Ja wârâ, was natürlich mit dem Gujarati-Bhili-Wort $\mathrm{Z}$ wârâ identisch ist.

Ein Zauberer (in diesem Gebiete Panda genannt), namens Phulsa und im Dorfe Batondha wohnend, setzte dort im Mai des Jahres 1939 ein Jawârâ-Fest in Szene. Leider haben wir damals nicht daran gedacht, zu fragen, ob das auf Grund eines eigenen Gelübdes geschah. Die Gesamtlage der Dinge spricht jedenfalls für diese Annahme. In bezug auf alles Wesentliche gleicht die Novene der Gond jener der Bhil. Trotzdem zeigt die Veranstaltung der Gond ihr eigenes Kolorit.

Der Zauberer (Panda) der Gond sät heute, obwohl die Veranstaltung den Namen Ja wâ râ (Gerste) trägt, nur Weizen. Er sät also auch nicht Weizen und Gerste, wie der Bhil-Zauberer das tut. Unser Panda Phulsa hatte den Weizen in irdenen Töpfen und in Bambusständern (Abb. 1), die natürlich vorher mit Erde angefüllt waren, aufgezogen. In diesem Falle stammten ein Topf und ein Bambusständer von Phulsa selbst, etwa zehn weitere waren von anderen Familien beigestellt worden. Wenn der Panda in Krankheitsfällen konsultiert wird, gibt er nicht selten die Weisung, daß bei Gelegenheit der nächsten Jawârâ-Veranstaltung eine Beteiligung notwendig sei. Der Panda erhält dafür als Opfergabe eine Ziege oder ein Huhn. Panda Phulsa behauptete allerdings, daß

10 V. ELWIN: The Baiga. London 1938, S. 64. 
seine Mata, die natürlich mit der Kali-Durga identisch ist, keine blutigen Opfer von ihm wolle, sondern statt dessen nur die Darbringung von Kokosnüssen verlange. Das ist aber keineswegs bei allen Gond-Pandas so; bei Phulsa gibt die Tatsache nur einen stärkeren Grad der Hinduisierung zu erkennen. Er erklärte denn auch selber, $\mathrm{da}$ er die Muttergöttin direkt darum ersucht habe, von ihm keine blutigen Opfer zu verlangen, sondern mit Kokosnüssen zufrieden zu sein.

Die erwähnten Bambusständer zeigen gewissermaßen zwei oder auch drei «Stockwerke». Das wird dadurch erreicht, daß man die starke Rinde eines etwa armdicken Bambusstabes an verschiedenen Stellen aufsplittert und diese Splitter selbst jeweils zu einem trichterförmigen Gebilde zurechtformt, wobei weitere, und zwar dünnere Bambussplisse im Sinne der Querflechtung zu Hilfe genommen werden. Es ist mithin ein Stück Korbflechterarbeit, die hier vorliegt. Der Korbflechter besorgt sie gegen ein geringes Entgelt. Töpfe und Bambusständer hatte Panda Phulsa in einem kleinen Anbau seines Hauses stehen, der, wie Dreizack und andere Dinge zeigten, überhaupt seine Kultstätte bildete.

Panda Phulsa trägt während der neun Jawârâ-Tage einen eisernen Ring um den Hals. (Abb. 2: Die «reitende» Person ist der Panda.) Das tut er so, weil auch die Kali Mata von' Kâlîghât bei Calcutta mit einem solchen Ring ausgestattet ist. Gleichzeitig hat Phulsa an einer Halsschnur eine durchbohrte Frucht hängen. Der Sadhu habe ihm dieselbe umgelegt. Am achten Tage der Novene wird die Opferung von Broten vorgenommen. Am neunten erfolgt die Hauptfeier. Gerade dieser konnte ich an Ort und Stelle beiwohnen.

Die Schlußfeier beginnt gegen vier Uhr nachmittags. Vor der Tür des Hauses des Panda Phulsa ist auf dem Boden mit Mehl ein Chauk gezeichnet (Abb.1). In der Nähe stehen drei Stangen, die vier bis sechs Meter hoch sind. Sie repräsentieren die Muttergöttinnen (Kali Mata usw.). An und für sich hätten hier sieben Stangen, für sieben Matas, zu stehen. Da Panda Phulsa aber erst seit einiger Zeit hier wohne, so erkläre das, wie versichert wurde, die noch unvollständige Einrichtung. $\mathrm{Zu}$ Füßen jener Matas, gleichzeitig im Angesichte des Chauk, liegt der Sohn des Panda, der offenkundig einmal dem Vater im Amte folgen soll, auf den Knien und bewegt (von der Mata "besessen») den Kopf rotierend hin und her (Abb. 3).

Alsdann folgt die Prozession zur Wasserstelle hin. Dabei spielt die Schwester des Panda eine Hauptrolle. (Abb. 2: Die Person links ist die Schwester des Panda.) Sie kann notfalls auch von einer anderen Frauensperson vertreten werden. Die Schwester des Panda trägt den Topf mit Weizen, den dieser selbst bereitgestellt hat. Vorher hat die Schwester den Bruder schon mit Milch und Gelbwurz-Tünche eingerieben; das besänftigt, wie gesagt wird, die sonst leicht aufgeregten Gottheiten.

Ein jüngerer Mann trägt nach Art einer Standarte die Stange, welche die MarhaiMata ${ }^{11}$ darstellt. Um zu bewirken, daß der Träger dieser Stange von der Marhai-Mata «besessen» wird, hat der Panda vorher Reiskörner gegen die Stange geworfen. Der Träger der Marhai-Mata-Stange zeigt sich denn auch rasch recht unruhig und läuft damit, als wenn eine geheimnisvolle Kraft ihn triebe, ruckweise hierhin und dorthin. Das Stangenende droht mehrere Male - es sieht so aus, als wenn eine unsichtbare Macht dazu zwänge - mit dem Boden in Berührung zu kommen. In solchen Momenten läßt vor allem die Frau des Panda ein großes Geschrei ertönen. Eine derartige Berührung darf nämlich nicht stattfinden. Es müßte sonst speziell über das Haus des Panda allerlei Unglück kommen.

Die Prozession hat die nächste, etwa tausend Meter entfernt liegende Wasserstelle zum Ziel. Abgesehen von dem Topf, den die Schwester des Panda trägt, werden die übrigen Töpfe und Bambusständer verteilt. Die Jugend vor allem steht mit hilfsbereiten

12 Die Marhai-Mata ist eine der sieben Matas des Panda. Es scheint, daß gerade ihr Pfahl gernc mit Pfaucnschwanzfedern geschmückı wird. An und für sich gilt die Marhai-Mata als cine gute Göttin. 
Händen zur Verfügung. Auf einmal schwingt sich der Panda auf die Schultern eines kräftigen, noch jüngern Mannes. Er reitet darauf, wie auf einem Pferd (Abb. 2). Wie er später erzählte, habe ihm die Mata plötzlich eingegeben, auf den Rücken jenes Mannes hinaufzusteigen. Auf die Frage, was wohl geschehen wäre, wenn die Wahl eine schwächere Person getroffen hätte, antwortete er schnell und bestimmt, daß das gar nichts gemacht hätte; denn die Mata wäre dem in einem solchen Falle gewiß mit der notwendigen Kraft zu Hilfe gekommen. Jener Träger wird übrigens für seine Mühewaltung nach-

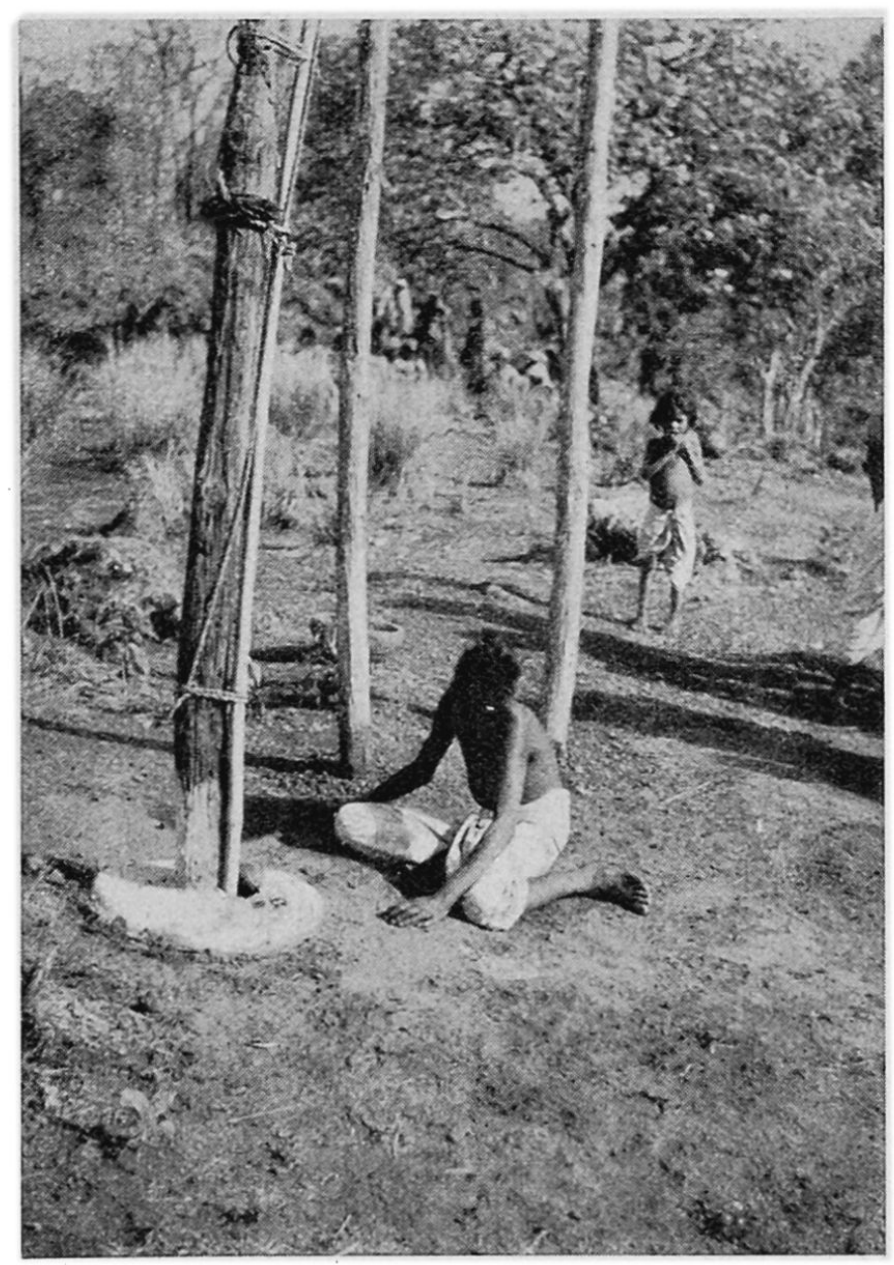

Abb. 3. Gond. Der Sohn des Zauberers liegt vor den Stangen, die die Matas repräsentieren. Photo Koppers

träglich mit einer Kokosnuß entlohnt. Zum Glück für ihn handelte es sich beim Panda Phulsa um eine schmächtige und leichte Person.

Etwa zweihundert Meter von der Wasserstelle wird für drei bis fünf Minuten gepaust. Man benützt die Pause, um schnell ein kleines Rauchopfer darzubringen.

An der Wasserstelle angekommen, nehmen alle Teilnehmer einige von den zarten Weizenpflänzchen und stecken sie sich hinter die Ohren. Alles übrige, also die Pflänzchen sowohl wie die anhaftende Erde, wird in das Wasser hineinversenkt. Dabei schont man aber sorgfältig die irdenen Töpfe, man nimmt sie nachher mit nach Hause, um sie dem praktischen Leben wieder dienstbar zu machen. Da man aber für die Bambusständer eine profane Verwendung nicht kennt, so läßt man sie nach erfolgter Entleerung an Ort und Stelle einfach liegen. Es stößt auf keinerlei Schwierigkeiten, als ich sie für meine Sammlung aufheben und mitnehmen lasse.

Mit einer allgemeinen Begrüßung (Umarmung) schließt die Jawârâ-Veranstaltung. 
Als Zweck der Uebung wird zusammenfassend angegeben: Die Mata soll alle und alles schützen und in Gesundheit und Wohlergehen erhalten, wachsen und gedeihen lassen. Die Gond wissen also darum, daß es sich bei der Veranstaltung um einen Fruchtbarkeitsritus handelt.

\section{Die Zusammengehörigkeit der Kali-Durga-Novene der Bhil und der Gond}

$\mathrm{Daß}$ es sich bei der Kali-Durga-Novene der Bhil und der Gond im wesentlichen um die gleiche Sache handelt, ist evident. In beiden Fällen steht eine neuntägige Veranstaltung, ein Fruchtbarkeitsritus, in Frage, den der Zauberer zu Ehren der großen Göttin Kali-Durga durchführt. Selbst in der Bezeichnung der Veranstaltung, Zwârâ (Jawârâ), was Gerste bedeutet, stimmt man überein. Das ist deshalb von Bedeutung, weil heute bei dieser Gelegenheit tatsäcblich entweder überhaupt keine Gerste, sondern nur Weizen gesät wird (so bei den Gond), oder eine Mischung von Gerste und Weizen das Saatgut bildet (so bei den Bhil). Da wir wissen, daß die Gerste dem Weizen gegenüber die ältere Getreideart darstellt, legt der erwähnte Sachverhalt die Annahme nahe, daß jener Fruchtbarkeitsritus bereits in der Gersten-Aera der südwestasiatischen Menschheit ins Leben trat. Sein relativ hohes Alter wäre damit gesichert.

Auf den ersten Blick könnte es scheinen, als widerstreite diese Feststellung der an anderer Stelle von uns vertretenen These, daß das heute Indien weithin beherrschende Zauberwesen starke Abhängigkeiten vom hinduistischen Tantrismus-Saktismus un$z w e i f e l h a f t$ hervortreten lasse ${ }^{12}$. Aber diese Abhängigkeit schließt anderseits das Vorhandensein und Fortbestehen alter Elemente in diesem Bereiche nicht aus. Daß es im Fruchtbarkeitsritus, den wir bei den Bhil und Gond kennenlernten, an Anklängen an ältere Vorbilder, ja an solche der Induskultur, die um 2500 vor Christi im nordwestlichen Vorderindien blühte, tatsächlich nicht fehlt, hoffe ich im folgenden zeigen zu können.

\section{Beziehungen zur Induskultur?}

$\mathrm{DaB}$ in der Induskultur Verhältnisse gegeben waren, in derien ein Fruchtbarkeitsritus, wie wir in der Kali-Durga-Novene der Bhil und der Gond kennenlernten, hätte existieren können, läßt sich unschwer wahrscheinlich machen.

Alles deutet darauf hin, daß die Induskultur bereits eine fortgeschrittene Stufe des Ackerbaues und der Viehzucht (im besonderen der Rinderzucht) erreicht hatte. Die Existenz des Pfluges ist zwar bis heute positiv nicht erwiesen, aber daß man eine Anschirrung des Rindes kannte, wird durch das Vorhandensein verschiedener Indizien nahegelegt. Darauf hat vor allem der bekannte Tierzuchtforscher W. AmschleR aufmerksam gemacht. So weist er hin auf die "girlandenartigen Gewinde um den Hals ", auf «das zwischen den Vorderbeinen hindurchlaufende Band, das um die Schulter läuft... und offenbar auf dem Rücken zusammengebunden war», Bänder, die beim sogenannten «Einhorn» als schabrackenartige Schulterverzierungen erscheinen ${ }^{13}$, usw. (Abb. 4).

An Getreidearten sind Gerste (Hordeum vulgare) und Weizen (Triticum sphaerococcum) belegt. Daß endlich die Muttergöttin mehr oder weniger im Zentrum des religiösen Kultes der Träger der Induskultur stand und daß sie eine der Wurzeln für den heute in Indien so weit verbreiteten Mata-Kult darstellt, kann als jetzt allgemein herrschende und wohl begründete Auffassung der maßgebenden Forscherkreise hingestellt werden ${ }^{14}$.

\footnotetext{
18 W. KOPPERS: Probleme der indischen Religionsgeschichte. Anthropos, 35/36, 1940/41, S. 704-814.

13 Nach einem bis jetzt unveröffentlichten Manuskript "Das Hausrind von Mohenjo-Daro", das mir sein Verfasser, Prof. W. AMSCHLER, in verdankenswerter Weise zur Verfügung stelite.

14 Hierzu z. B. H. MODE: Indische Frühkulturen und ihre Beziehungen zum Westen. Basel 1944; W. KOPPERS (Anm. 12). Der. selbe, Zum Ursprung des Mysterienwesens im Lichte von Völkerkunde und Indologie. Eranos-Jahrbuch 1945, 11, Zürich, S. $215-275$.
} 


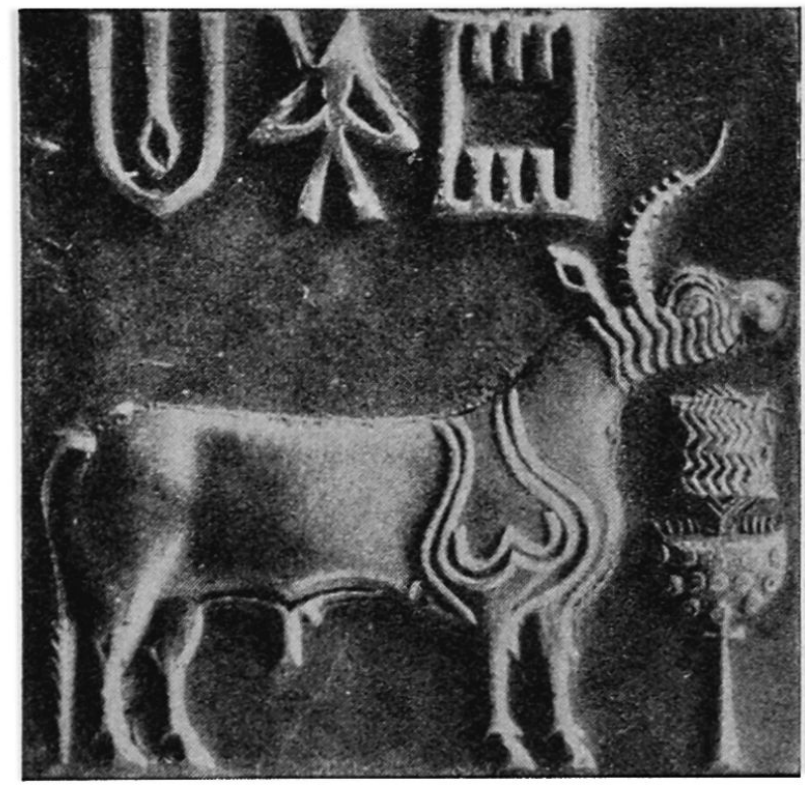

Abb. 4. Induskultur. Primigenius-Rind mit Anzeichen einer Anschirrung. (Marshall, III, Tafel C IV, 38)

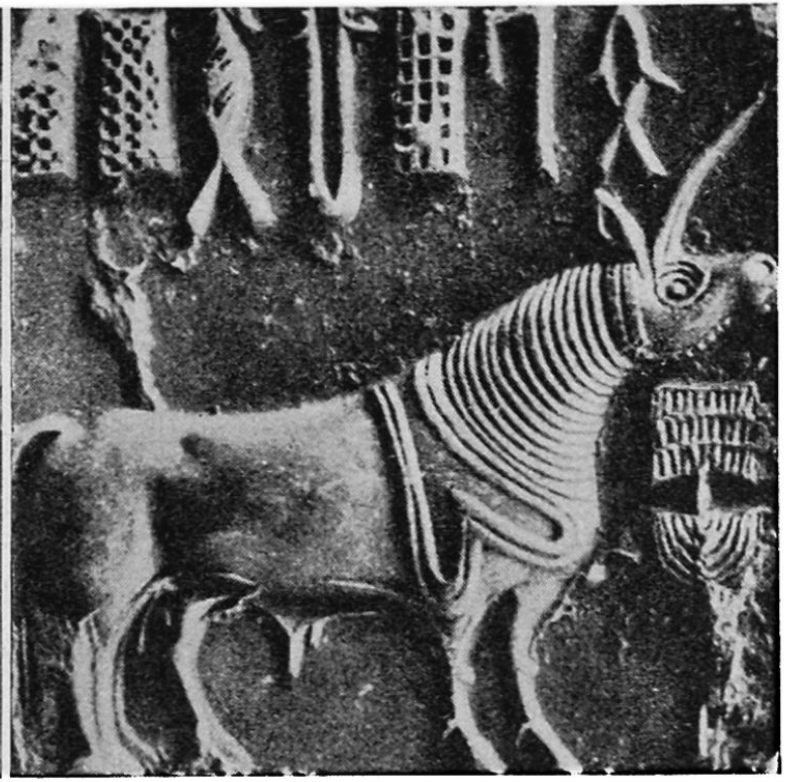

Abb. 5. Induskultur. Primigenius-Rind mit Ringen um den Hals. (Marshall, III, Tafel C III, 4)

Aber die Frage stellt sich, ob auch im Konkreten und Einzelnen Beziehungen zwischen der Kali-Durga-Novene, wie wir sie bei den Bhil und Gond kennenlernten, und der Induskultur festgestellt oder doch wahrscheinlich gemacht werden können. Ich glaube zeigen zu können, daß dieser Fall gegeben ist. Interessanterweise haben wir es dabei mit einem Gegenstand der Induskultur zu tun, der ihren Erforschern und Erklärern schon viel Kopfzerbrechen bereitet und eine gute Anzahl von wenig befriedigenden Theorien entlockt hat.

Bekanntlich spielt auf den Siegeln der Induskultur die Darstellung des Rindes eine große Rolle. Viele Siegel zeigen die Darstellung des Bos taurus namdaicus, also des Ur oder des Primigeniusrindes. Zwei Eigentümlichkeiten zeichnen diese Darstellung aus. Die eine besteht darin, daß das Tier stets nur einhörnig wiedergegeben wird, man hat deshalb auch von ihm als dem Unicornis (einem Fabeltier) gesprochen und geschrieben. Wie W. AMSCHLER wahrscheinlich gemacht hat, müssen es zeichnerische Schwierigkeiten gewesen sein, die die Künstler veranlaßten, dieses Tier immer nur so zur Darstellung zu bringen ${ }^{15}$. Es dürfte ihnen zu große Schwierigkeiten bereitet haben, die eigentümlichen Windungen der beiden Hörner befriedigend wiederzugeben, und so wurde als Ausweg die schematisierte Darstellung nur eines Hornes, und zwar in Seitenansicht, gewählt. Das zweite Horn hätte man sich also genau dahinterstehend zu denken. Ob diese Erklärung die wirklich zutreffende ist oder nicht, können wir hier dahingestellt sein lassen. Es ist wichtig, daß auch immer nur ein Ohr des Tieres wiedergegeben ist. Für unsere weiteren Darlegungen kommt diesem Punkt keine besondere Bedeutung zu. Jedenfalls lassen die im übrigen eindeutigen Kennzeichen des Bos taurus namadicus, wie AMSCHLER und FRIEDRICHs feststellten, die Annahme, daß es sich hier, wie einzelne Forscher meinten, um ein Fabeltier handle, als ausgeschlossen erscheinen.

Die zweite Eigentümlichkeit, welche der Bos taurus namadicus auf den Siegeldarstellungen aufzuweisen hat, ist mit der Tatsache gegeben, daß das Tier ausnahmslos seinen Kopf ausgestreckt hält über ein Gestell, dessen Bedeutung bis heute eine sehr umstrittene ist ${ }^{16}$. So wurde es von MarShall für ein Räuchergefäß gehalten ${ }^{17}$. Fried-

\footnotetext{
1s W. AMSCHLER (Anm. 13). Zur Charakterisierung des «Einhorn»-Rindes vgl. auch H. F. FRIEDRICHS, Dic Kenntnis der frühgeschichtlichen Tierwclt Südwestasiens. Leipzig 1933.

10 Bemerkenswerterweise erscheint dieser Gegenstand nur in Verbindung mit dem «Einhorn». Es ist nur cine Ausnahme bekann geworden, dic aber nacb MARSHALL "appears to be mercly a variant of the unicorn type». (Anm. 1, S. 69).

17 E. MARSHALL (Anm. 1), S. 69.
} 
RICHS glaubte, darin eine Verbindung von Raufe und Krippe erblicken zu müssen ${ }^{18}$. H. Mode denkt an einen Altar ${ }^{\mathbf{1}}$. Auch die Meinung, daß ein Vogelkäfig in Frage stehe, ist geäußert worden ${ }^{20}$.

Als ich im Mai 1939 bei den Gond an der Schlußfeier der Durga-Novene teilnehmen konnte und bei der Gelegenheit die bereits oben erwähnten Bambusständer mit dem jungen Weizenpflänzchen darin erblickte, erinnerte ich mich gleich jenes mysteriösen Gegenstandes der Induskultur. Da mir an Ort und Stelle die Literatur nicht zur Hand war, mußte die genauere Ueberprüfung der Angelegenheit vertagt werden.

Am zutreffendsten hat, soweit ich es zu beurteilen in der Lage bin, E. MACKAY den Charakter des kritischen Objektes bestimmt. MACKAY hat bekanntlich im Jahre 1938 zwei weitere Bände des großen Mohenjo-Daro-Werkes herausgegeben. Es erscheint daher wohl verständlich, $\mathrm{da} \beta$ er, von den früheren Arbeiten und Publikationen profitierend, im Einzelfall die am meisten ansprechende Deutung zu bieten in der Lage ist ${ }^{21}$.

Wenn MaCkay in unserem Gegenstand ein Wickerwork-Produkt, also ein Produkt der Flechtarbeit ${ }^{22}$, erblickt, so ist sicher, daß diese Bestimmung für den größten Teil der Fälle als die am meisten ansprechende und zutreffende erscheint. Es spricht meines Erachtens auch nichts dagegen, in bezug auf das Material jener Gegenstände in erster Linie an Bambussplissen zu denken. Der allgemeinen Zustimmung sicher ist ferner auch Mackays Deutung, der gemäß das Objekt dem Kultbereiche angehört. Das legt nicht nur die Darstellung nahe, die hier zunächst in Frage steht, also die Darstellung in der charakteristischen Verbindung mit dem Bos taurus namadicus, sondern auch die Tatsache, daß das Objekt (in vergrößerter Form) auch als selbständiger Kultgegenstand identifiziert werden kann, der zudem in einer Prozession mit herumgeführt wird ${ }^{23}$. Es ist ferner E. MACKAY gewesen, der, soweit ich sehe, als erster darauf hingewiesen hat, daß der Gegenstand an und für sich nicht fest steht, sondern, soll er nicht umfallen, gehalten (in den Boden gesteckt?) oder getragen werden muß. Flechtwerk, Kultgegenstand und Verwendung bei Gelegenheit einer Prozession, in bezug auf alle diese Punkte wäre, wie man sieht, die Uebereinstimmung mit den Bambusständern der Gond vollauf gegeben.

Aber die Uebereinstimmung wäre eine noch frappantere und schlagendere, wenn man zeigen könnte, daß jene Gegenstände auch von den Leuten der Induskultur zum (kultischen!) Anbau von Getreidepflänzchen benutzt wurden. Jedenfalls in drei Fällen zeigen die Siegeldarstellungen ein Bild, das diese Annahme, wie mir scheint, sehr nahe legt. Jene strahlenförmigen Gebilde, die MARShaLl als Feuerflämmchen deuten zu müssen meinte (Abb. 4), erklären sich gewiß viel zwangloser und natürlicher, wenn man sie als Getreidehälmchen gelten läßt ${ }^{24}$. Ein Vergleich mit den Bambusständern der Gond wirkt da direkt überraschend.

Eine Schwierigkeit gegen diese Auffassung könnte indes in dem Umstand gesehen werden, daß nur in den genannten drei Fällen jene Hälmchen zu erkennen sind, während das für die große Zahl der anderen Darstellungen des gleichen Gegenstandes nicht zutrifft. Vielleicht darf aber die Eirklärung hierfür darin gesehen werden, daß die Wieder-

18 Vgl. E. J. H. MACKAY; Further Excavations at Mohenjo-Daro. I. New Delhi 1938, S. 670. MACKAY bezieht sich dabei auf die unter Anm. 15 genannte Arbeit von FRIEDRICHS.

10 Anm. 14, S. 56.

so Siehe bei H. MODE (Anm. 14), S. 55.

21 E. MACKAY (Anm. 18), S. 670.

22 E. MACKAY: Excavations at Chanhu-daro by the American School of Indic and Iranian Studies and the Muscum of Finc Arts. Boston: Season 1935-1936. Bulletin of the Museum of Finc Arts. Boston 34, 1936, S. 83-92. Siche S. 89.

${ }^{23}$ E. MACKAY (Anm. 18), S. 670. «I do not think, howerer, that this particular object can be so casily explaircd, becausc, as I have pointed out elsewhere, it is carricd in procession, not only with the animal with which it is always associated but even by itself, on certain amulets from Harappa." Vgl. die Abb. bei MARSHALL: I. Tafel XIII, 18, 19, 20. Und MARSHALL: III. Tafel C XVI, 5, 8.

3t Dem in Abb. 4 wiedergegebenen Beispiel gleichen im wesentlichen zwei weitere, nämlich MARSHALL I, Tafel XIII, 22 und II, Tafel CII, c. 
gabe in den allermeisten Fällen ziemlich dunkel und schematisch gehalten ist. Wo die Hälmchen sichtbar werden, handelt es sich um besser erhaltene (oder überhaupt um von Haus aus sorgfältiger gearbeitete) Exemplare.

Die weitere Frage, ob jene Gegenstände bei den Leuten der Induskultur auch eine profane Aufgabe zu erfüllen hatten, vermögen wir mit Sicherheit nicht zu beantworten. $\mathrm{Daß}$ die Gond für ihre Bambusständer eine praktische Verwertung nicht kennen, haben wir gesehen. Dieser Sachverhalt spricht wohl dafür, daß sie, wenn nicht den ganzen Ritus, so doch dieses Detail daraus einmal von irgendwoher übernommen haben. $\mathrm{Ob}$ dafür die Induskultur als direkte Quelle in Betracht käme, ist natürlich wenig wahrscheinlich. Aber an die Induskultur als indirekte Quelle zu denken, dürfte als gestattet erscheinen.

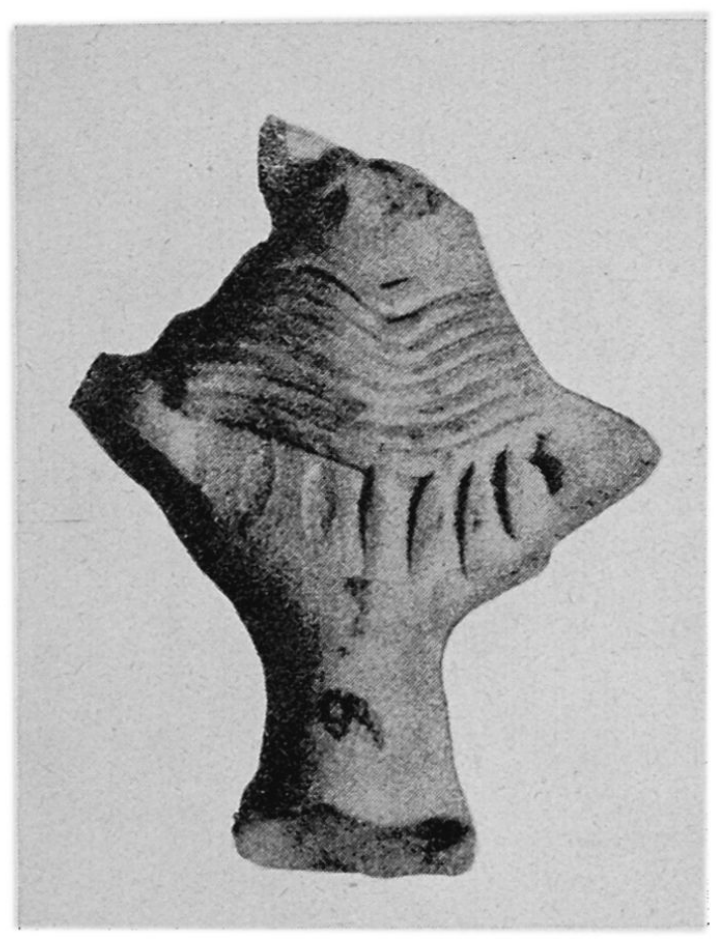

Abb. 6. Induskultur. Fragment der Darstellung eines weiblichen Idols (Muttergöttin?), mit Ringen um den Hals. (Marshall, I, Tafel XII, 8.)

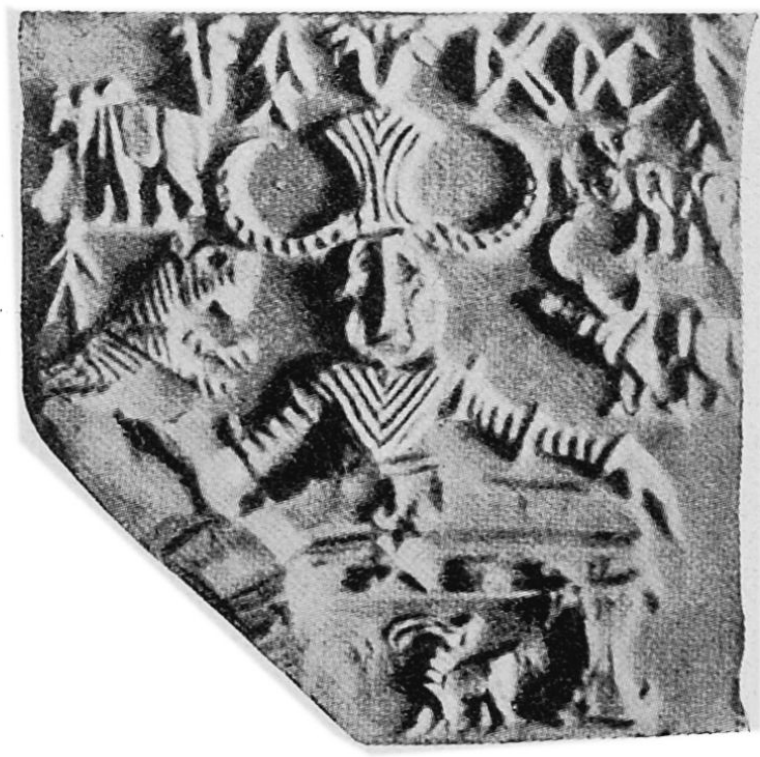

Abb. 7. Induskultur. Thronender Gott (Proto-Siva?), mit Ringen um den Hals. (Marshall, I, Tafel XII, 17.)

Eine Andeutung dafür, daß unser Gegenstand bzw. der Stier, mit dem er immer wieder in besonderer Weise verbunden erscheint, auch nach außen hin erkennbare Beziehungen zur Muttergöttin und zu ihrem Trabanten, dem «three-faced God» (Proto-Siva) hervortreten läßt, darf vielleicht in dem gleichen oder doch ganz ähnlichen Halsschmuck gesehen werden, der allen dreien, also dem «Einhorn»-Stier (Abb. 5), der Muttergöttin (Abb. 6), und dem Proto-Siva (Abb. 7), eigentümlich ist. Auf diese Tatsache hat seinerzeit schon J. Marshall hingewiesen ${ }^{25}$. Wir erinnern uns hier des Eisenringes, den der Gond-Panda zur Zeit der Novene um den Hals trägt, und zwar mit Rücksicht auf die Kali-Durga von Kâlîghât bei Calcutta, die ebenfalls mit einem derartigen Ring ausgestattet sein soll.

Trotz allem möchte ich in bezug auf das erörterte Problem nicht mehr behaupten, als die Voraussetzungen angesichts des gegenwärtigen Forschungsstandes gestatten. Von einer vollen Sicherheit des Zusammenhanges zwischen den Gond und der Induskultur kann wohl nicht gesprochen werden, aber eine gut begründete Wahrscheinlich-

25 MARSHALL (Anm. 1), S. 69. 
keit ist gegeben. Die Aufgabe weiterer Forschung wird es sein, die Angelegenheit einer befriedigenderen und eindeutigeren Klärung zuzuführen ${ }^{26}$.

$\mathrm{Da}$ es auch sonst in der indischen Bevölkerung an Anzeichen für Verbindungen mit dem alten vorindogermanischen Orient nicht zu fehlen scheint, mag noch folgendes Beispiel zeigen. In dem Dorfe Aulia unweit Khandwa (Central Provinces) leben hauptsächlich Angehörige hinduistischer Ackerbaukasten (Kunbi, usw.). Hier werden bei Gelegenheit eines zu Ehren der Muttergöttin veranstalteten Fruchtbarkeitsfestes, wie mir von dem an Ort und Stelle weilenden Missionar K. Schmint in dankenswerter Weise erzählt wurde, tischartige Gestelle umhergetragen, auf denen ziemlich roh geformte weibliche Figuren, etwas unter Lebensgröße, in stehender Haltung befestigt

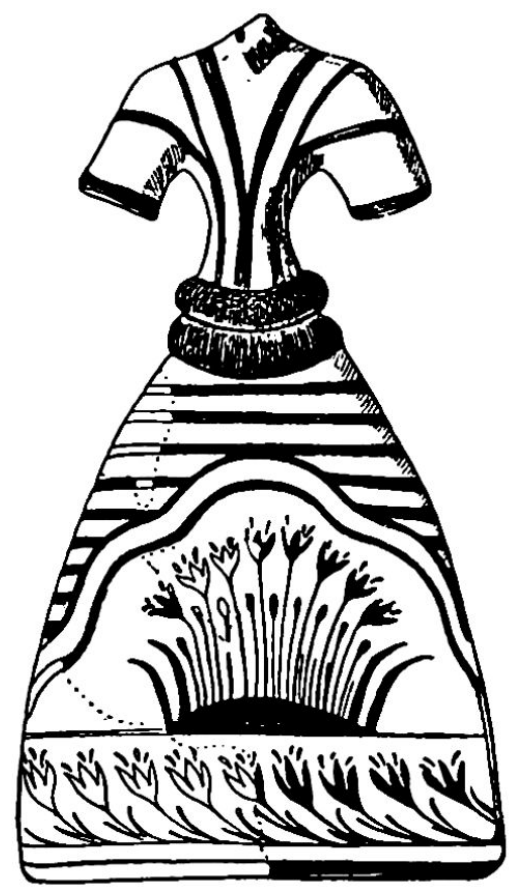

Abb. 8. Alt-Kreta (Knossos). Weibliche Gestalt (Muttergöttin?). Unten Pflanzen-und Blumenarrangement. (Nach C. Clemen, Religionsgeschichte Europas I, Heidelberg 1926, S. 102.)

sind. Vorne auf das Gestell, zwischen die Füße der Figur, unter die herunterhängenden Kleider, mit denen sie ausgestattet ist, wird das Körbchen mit dem jungen Weizen hingestellt. $\mathrm{DaB}$ wir es hier mit dem im Grunde gleichen Ritus zu tun haben, den wir in der Kali-Durga-Novene der Bhil und Gond kennenlernten, kann nicht bezweifelt werden. Aber weiterhin denkt man dabei unwillkürlich an die bekannte Darstellung von Knossos, die eine weibliche Gestalt zeigt, deren Kleid unten ausgespart und dafür mit einem Pflanzen- und Blumenarrangement ausgestattet ist ${ }^{27}$, das schon früher, gewiß mit Recht, im Sinne eines Fruchtbarkeitsritus gedeutet worden ist (Abb. 8). Die Uebereinstimmung zwischen Alt-Kreta (Knossos) und dem heutigen bäuerlichen Indien könnte, was den vorliegenden Fall anbetrifft, kaum schlagender sein.

Als Verbindungsbrücke kommt sehr wahrscheinlich auch in diesem Falle - dafür spricht jedenfalls die Gesamtlage der Dinge - der Komplex der Induskultur in Betracht. In bezug auf die Interpretation ihrer Elemente sind wir, wie in andern ähnlichen Fällen, solange die Schrift nicht lesbar ist, vielfach behindert. $\mathrm{Daß}$ die ethnologische Forschung

20 Uebrigens ist es wohl nicht ausgeschlossen, daß die von Assam bis ins östliche Indonesien verbreiteten, aus Bambus verfertigten trichterförmigen Opferkörbe in die gleiche Reihe mit unseren Bambusständem usw. gehören (Heinc-Geldern in Buschans «Illustr. Völkerkunde", II, Stuttgart 1923, S. 860). Sie dienen "als eine Art Altar, bei den Wa in Birma, den Kalinga und Tinggian in Nord-Luzon und den Batak in Sumatra auch zur zcitweiligen oder dauernden Aufnahme der auf der Kopfjagd erbeuteten Schädel".

27 C. CLEMEN: Religionsgeschichte Europas I, Heidelberg 1926, S. 102, Abb. 85. 
mit berufen und befähigt ist, Licht in das hier noch obwaltende Dunkel hineinzubringen, glaube ich mit meinen Ausführungen gezeigt zu haben. Dieses Bemühen gewinnt an Bedeutung, wenn dabei Lichtstrahlen nicht nur auf Probleme der Induskultur, sondern weiterhin auch auf solche der Antike fallen; denn damit rühren wir an die Grundlagen, näherhin an die vorindogermanischen Grundlagen, unserer eigenen europäischen Kultur. $\mathrm{Da}$ vor allem der von Indien ausgehende Völkerforscher zur Aufhellung dieser ebenso interessanten als bedeutungsvollen Fragen sein Scherflein beizutragen in der Lage ist, ist nicht sein eigenes Verdienst. Vielmehr hängt das mit der bekannten Tatsache zusammen, daß dort noch heute vieles von dem lebendige Wirklichkeit ist, was sonstwo seit Jahrtausenden im Schmelztiegel der höheren Kulturentwicklung, sei es weitgehend umgestaltet, sei es völlig, ausgemerzt wurde.

\section{DIE TÄTIGKEIT DER ARBEITSGEMEINSCHAFT ZUR GEOMOR- PHOLOGISCHEN ERFORSCHUNG DER SCHWEIZER ALPEN 1945}

Die Arbeit der AGES. ist erfreulicherweise auch im Jahre 1945 gefördert worden. Von ihren bisherigen Resultaten zeugen mehrere im Schweizer Geograph XXII, 1945, publizierte Aufsätze, die das Bestreben erkennen lassen, die Sachforschung mit methodologischer Diskussion zu verbinden, woraus zweifellos die optimalste Klärung der Probleme zu erwarten ist. Die beiden nachfolgenden Berichte über die offiziellen Anlässe der Arbeitsgemeinschaft im vergangenen Jahre erweisen, daß diese auf erfolgverheißendem Wege marschiert.

1. Die Exkursion ins Maderanertal vom 18./19. August 1945.

Ziel der von Dr. E. SCHWABE und PD. Dr. W. STAUB geleiteten Exkursion war die Untersuchung der morphologischen Probleme des Tales und die Diskussion über die Methoden der geomorphologischen Kartierung.

Nach kurzer Orientierung STAUBs über die geologischen Verhältnisse des Gebietes erreichten die Teilnehmer von der Bahnstation Amsteg-Silenen aus, anfangs dem alten Gotthardweg folgend, den Rundhöcker, auf dem die Ruine Zwing-Uri steht (P. 578). Hangwärts, durch eine Erosionsrinne von ihm getrennt (abwechselndes Vorrücken und Abschmelzen des Gletschers), lehnt sich ein wahrscheinlich dem Gschnitzstadium zuzuordnender Moränenwall an, der sich, gekennzeichnet durch Gletscherschliffe und Moränenblöcke, bis nach Frenschenberg (P. 816) verfolgen läßt. Die von hier aus schön überblickbaren Verhältnisse der Konfluenz von Reuß- und Maderanergletscher erweisen sich als recht kompliziert. Der unterste Boden (um Ried, P. 678) oberhalb der Haupttalstufe dürfte dem Talboden von Bristen $(723 \mathrm{~m})$ genetisch entsprechen (Würm?). Unterhalb der Stufe ist die Felssohle unter Alluvium verborgen. Die Bildung des Haupttales der Reuß von Amsteg talabwärts scheint alt zu sein, wie die interglazialen Bergsturzreste von Schattdorf-Haldiberg aus der letzten Zwischeneiszeit beweisen.

Weitere, noch nicht sicher zu klassierende Niveaureste finden sich bei Frenschenberg $(880 \mathrm{~m}$, $960 \mathrm{~m}$ ); Waldiberg (dürfte mit $1360 \mathrm{~m}$ der schönen, wahrscheinlich präglazialen Arnibergterrasse von $1380 \mathrm{~m}$ entsprechen); Rückenegg und Sonnengrat (gehören mit ca. $1900 \mathrm{~m}$ zu deri Niveauresten eines der höchsten Verflachungsgebiete). Nach SCHWABE lassen sich diese Terrassen mit den verschiedenen Leisten im Maderanertal in folgende Niveaus einordnen:

1. Rückenegg . . . . . . . . . Stafelalpen (1900 m) - Alp Gnof (1925 m) - Düssialp $(1959 \mathrm{~m})$.

2. Waldiberg (vermutlich präglazial) . . Geschel $(1520 \mathrm{~m}) \cdot$ - Tritt $(1642 \mathrm{~m})$ - Hüfigletscher (unterhalb P. 1971).

3. Zwischenniveau . . . . . . . Griessernalp $(1305 \mathrm{~m})-$ Balmenegg (1351 m) - Sassegg (ca. $1500 \mathrm{~m}$ ).

4. Frenschenberg . . . . . . Lungenstutz $(1100-1140 \mathrm{~m})$ - Sanderfeld vor Hüfigletscher (ca. $1400 \mathrm{~m})$.

Zur Höhe der aus amphibolitischen Gesteinen bestehenden Terrassenbastion "Auf der Breitlaui» (P. 1133 S. Bristen) zieht von E her eine prächtige, in den weichen Karbonschichten angelegte Verwitterungsterrasse hinauf. Auch in der engen Steilstrecke des Maderanertobels prägt sich der Geschel als Amphibolithärtling aus. Verschiedene wallmoränenartige Bildungen beidseits des Kärstelenbaches im Tobel und oberhalb verlangen genaue Untersuchung (schöner Endmoränenbogen im Balmenwald SW Hotel SAC.).

Während SCHWABE nach dem Nachtessen einen Ueberblick über die Morphologie des Tales gab, wurden während des regnerischen Sonntagvormittags bis $10 \mathrm{Uhr}$ Einzelfragen der morphologischen Kartierung diskutiert.

Bei aufhellendem Wetter zeigte eine Wanderung gegen $\mathrm{Sa}$ (prächtige Stromquelle des auf der Alp Gnof versickernden Wassers; Bergsturzsee von Butzli ohne oberirdischen Abfluß) einen aufschluß- 